'Grupo de Investigación en Odontogeriatría (GIOG), Escuela de Odontología, Universidad de Talca. Talca, Chile.

${ }^{2}$ Programa de Investigación en Excelencia Interdisciplinaria en Envejecimiento Saludables (PIEI-ES), Universidad de Talca. Talca, Chile. ${ }^{a}$ Cirujano-Dentista. ${ }^{\mathrm{b}} \mathrm{MSc}, \mathrm{PhD} \odot$. CPhD.

Este estudio fue financiado por el Proyecto Fondecyt 1140623 de RAG y con Fondos del Programa PIEI-ES de la Universidad de Talca de SL.

Recibido el 9 de abril de 2015, acepado el 1 de diciembre de 2015.

Correspondencia a: Dr. Rodrigo A. Giacaman, PhD. Universidad de Talca 2 Norte 685 Teléfono: (+56-71-2201546) giacaman@utalca.cl

\section{Realidad y desafíos de la salud bucal de las personas mayores en Chile y el rol de una nueva disciplina: Odontogeriatría}

\author{
SORAYA LEÓN ${ }^{1,2, a, b}$, RODRIGO A. GIACAMAN ${ }^{1,2, a, c}$
}

Reality and challenges of the oral health for older adults in Chile and the role of a new discipline: geriatric dentistry

Chile is experiencing one of the fastest aging processes in Latin America. The implications derived from this phenomenon involve many aspects of the society, especially health care. In particular, insufficient oral health coverage in the country limits oral care provision for a population with a high prevalence and severity of oral diseases. These conditions include dental caries, periodontal disease, tooth loss, defective prostheses, oral mucosa lesions and xerostomia, among others, and strongly affect quality of life of the elderly population. Furthermore, dental curriculum of most dental schools lack specific training of students in geriatric dentistry or gerodontology. Hence, newly graduated professionals are not competent to satisfy the needs of this growing and increasingly demanding population of older adults. Within this demanding context, Chile may find the potential to become a model and referent to deal with the challenge, incorporating innovative changes in education and public health strategies for the older population by an interdisciplinary approach.

(Rev Med Chile 2016; 144: 496-502)

Key words: Aging, Dental Caries, Chile, Education, Dental, Geriatric Dentistry, Oral Health.
Envejecimiento poblacional y su impacto en salud

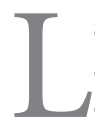

a población mundial está envejeciendo, siendo los grupos de mayor edad los que más crecen proporcionalmente ${ }^{1}$. A pesar de que estos cambios demográficos tuvieron su momento más dramático en la última parte del siglo XX y durante la primera parte del siglo XXI, se espera que la tendencia continúe e incluso se incremente en el futuro ${ }^{2}$. América Latina, igualmente, se encuentra en medio de esta transformación demográfica de largo alcance. En las décadas pasadas, la natalidad ha caído dramáticamente en casi toda la región y hoy se encuentra cerca, o incluso por debajo, de la tasa de reemplazo de 2,1 en la mayoría de los principales países de la región ${ }^{3}$. Tal es la situación que se estima que algunos países latinoamericanos como Brasil, Chile y México, podrían llegar a tener poblaciones más envejecidas que la de los Estados Unidos de Norteamérica. Es interesante notar que no obstante tanto países desarrollados como aquellos en desarrollo han envejecido o están envejeciendo. Los primeros se convirtieron en sociedades prósperas antes de envejecer, mientras que los segundos están o han envejecido sin alcanzar la prosperidad ${ }^{3}$.

Es evidente que envejecer en un contexto social próspero trae aparejadas una serie de ventajas que finalmente repercuten positivamente en la calidad de vida de la población mayor, a diferencia de lo que ocurre cuando el entorno social es limitado y 
vulnerable. Estos fenómenos sociales son nuevos para la humanidad y representan serios desafíos para la sociedad en su conjunto.

Chile no está al margen de esta realidad y en los últimos 30 años ha experimentado un proceso de envejecimiento demográfico acelerado sin precedentes históricos. Actualmente, Chile es considerado uno de los países con "envejecimiento avanzado" y se espera que para el año 2025 tenga el índice de envejecimiento más alto de la región, llegando a tener una proporción de personas mayores cercana a $28 \%$ para el año $2050^{4}$.

Como una forma de hacer frente a este panorama actual y previendo que la situación de envejecimiento seguiría una tendencia similar en los próximos años, Chile desarrolló una Política Integral de Envejecimiento Positivo para el período 2012-2025 . Esta política de Estado provee un marco normativo, a partir del cual se diseñan e implementan políticas públicas para el mejoramiento efectivo de la calidad de vida de las personas mayores.

En el área de la salud, Chile cuenta con un programa de salud para las personas mayores, pero desafortunadamente en el área odontológica es insuficiente. Actualmente, la cobertura odontológica para la población mayor abarca sólo atenciones de urgencia ambulatoria y atención integral odontológica exclusivamente para quienes tienen 60 años $^{6}$, dejando sin cobertura a los mayores de 60 años y a las personas con grados mayores de dependencia que les impide trasladarse hacia los lugares donde se provee de atención ambulatoria. Con la sola excepción de la fluoración del agua potable, que es una medida de cobertura universal, la mayoría de los programas odontológicos para la comunidad están intencionados para beneficiar casi exclusivamente a niños y adolescentes ${ }^{7}$. La mayor expectativa de vida de la población y la carencia de cobertura odontológica actual hará que se acrecienten las desigualdades entre los distintos grupos etarios. El Estado tiene así la tarea de implementar políticas públicas para acortar las brechas de acceso y beneficios entre la población mayor y los más jóvenes.

\section{Envejecimiento y salud bucal en personas mayores}

Las personas mayores presentan una gran variedad de problemas bucales tales como caries, enfermedad periodontal, pérdida dentaria, próte- sis dentales no funcionales, lesiones en la mucosa oral y xerostomía, entre otros. Estos problemas, a su vez, pueden impactar fuertemente su calidad de vida. A modo de ejemplo, la pérdida de dientes o la presencia de lesiones de caries extensas y dolorosas pueden afectar el estado nutricional de una persona mayor, toda vez que el trastorno bucal llevará a que seleccione alimentos blandos, usualmente de bajo valor nutritivo. Aspectos de la esfera sicosocial también pueden ser afectados por problemas bucales, dado que limitan la capacidad de hablar, sonreír y sociabilizar ${ }^{8}$. En otro ámbito, se ha descrito una asociación entre la salud bucal y varias enfermedades sistémicas debido a factores de riesgo comunes, como es el caso de la diabetes mellitus ${ }^{9}$, las enfermedades cardiovasculares ${ }^{10} \mathrm{y}$ respiratorias ${ }^{11}$, todas patologías de alta prevalencia en la población mayor. Además, las enfermedades crónicas limitan la tolerancia de las personas mayores a los procedimientos dentales o disminuyen su capacidad neuromuscular para adaptarse a los tratamientos rehabilitadores protésicos.

Actualmente, la mayoría de las personas mayores en los países industrializados conservan más dientes naturales ${ }^{12}$. Esto, debido entre otras razones a las mejoras en el acceso y condiciones sanitarias, al impacto del uso de los fluoruros y al desarrollo de una odontología más conservadora $^{13}$. La población mayor actual, a diferencia de sus predecesores, tiene mayor conocimiento y expectativas más altas. En ese sentido, uno de los aspectos más destacados es que las personas mayores que permanecen funcionalmente independientes buscan atención dental al igual que las personas más jóvenes dentadas ${ }^{14}$.

Si bien es cierto que los problemas bucales en este grupo etario son más complejos, la solución radical, basada en la extracción dentaria y una prótesis completa, ya no satisface las necesidades para estas personas que demandan la conservación de su remanente biológico ${ }^{15}$. El conservar los dientes naturales, un objetivo altamente deseable, implica al mismo tiempo mayor riesgo de padecer las enfermedades bucales más prevalentes como caries en la corona y raíces de los dientes y enfermedad periodontal ${ }^{16}$. El mayor riesgo se debe principalmente a cambios patológicos relacionados con la edad, como la recesión gingival y modificaciones en el microambiente bucal, los que se asocian, a su vez, a cambios en el biofilm microbiano que se deposita sobre los dientes, el cual muchas veces 
es difícil de eliminar de la superficie dentaria ${ }^{17}$. Así también, otra situación a considerar es el efecto que tienen los fármacos utilizados para el tratamiento de las enfermedades crónicas, muchos de los cuales tienen propiedades anticolinérgicas, produciendo hipofunción salival o xerostomía en aproximadamente $30 \%$ de la población mayor ${ }^{18}$. Una disminución en la calidad y cantidad de saliva provoca alteraciones en la masticación, degustación y deglución, incluso para el uso confortable de las prótesis dentales removibles. Debido a que la mayoría de las personas mayores utiliza este tipo de aparatos, también tienen mayor riesgo de presentar lesiones de la mucosa bucal asociadas a las prótesis. Por su parte, una prótesis mal ajustada o defectuosa repercute en patrones de alimentación y nutrición inadecuados ${ }^{19}$. Así, las personas mayores médicamente comprometidas con algún grado de dependencia funcional, ven limitado su acceso a la atención odontológica debido a barreras de tipo médico, socio-económico, psicológico, geográfico y educacional y en ese escenario la salud bucal puede impactar aun más en su salud general. La evidencia ha mostrado que una mala higiene bucal y la pérdida de dientes puede aumentar la morbilidad e incluso la mortalidad en personas mayores frágiles ${ }^{20}$.

\section{Indicadores de salud bucal en personas mayores}

Pese a la escasez de datos epidemiológicos recientes, es posible observar una mejoría en la salud bucal de la población chilena en el último tiempo. No obstante, este cambio parece no haber incluido a los adultos mayores. De acuerdo a la Encuesta Nacional de Salud de 200321, los desdentados parciales corresponden a $69,8 \%$ y los desdentados totales alcanzan cerca de $30 \%$ de la población general. Pese a lo alarmante de las cifras, sólo 37,1\% usaba prótesis en ambos maxilares y $25 \%$ sólo en el maxilar superior. La última Encuesta Nacional de Salud del $2009-2010^{22}$ por su parte, mostró que $22,9 \%$ de los encuestados usaba prótesis dental, de ellos, 15,5\%, correspondientes a los de 65 y más años, se manifiesta "poco o nada conforme" con ella. En este mismo rango etario, 55,3\% tenía la percepción de necesidad de uso de prótesis dental, $31,1 \%$ no ha visitado al odontólogo en los últimos 5 años y $4,3 \%$ nunca lo ha visto.
No muy diferentes son los resultados de un estudio realizado en la región del Maule ${ }^{23}$, donde $74,9 \%$ de las personas mayores correspondían a desdentados parciales y $25,1 \%$ restante a desdentados totales. El $72 \%$ empleaba al menos un aparato protésico, ya sea maxilar o mandibular, mientras que $28 \%$ restante señaló no utilizar ninguna prótesis. Además, el número de dientes remanentes promedio en los individuos dentados fue de 9,09 , muy por debajo de lo que se considera una oclusión funcional correspondiente a 20 dientes en contacto con sus antagonistas ${ }^{24}$. Este estudio mostró también que existían peores condiciones de salud bucal en la población rural en comparación con su contraparte urbana.

En este complejo escenario, los dentistas generales debidamente capacitados serán clave en la provisión de cuidados bucales para esta población envejecida con diferentes niveles de dependencia ${ }^{25}$, lo que impactará directamente en mejorar la salud bucal de esta población y por lo tanto su calidad de vida.

\section{Educación en odontogeriatría}

De la alarmante situación bucal descrita anteriormente, es claro que parte de las soluciones pasan necesariamente por una debida capacitación de los profesionales odontólogos. La formación tradicional en las escuelas de odontología de Chile no ha incluido la salud bucal de los mayores como uno de sus ejes curriculares.

La odontogeriatría puede ser definida como "aquella parte del plan de estudios odontológicos de pregrado que se ocupa del conocimiento específico, las actitudes y las competencias técnicas requeridas en la prestación de atención en salud bucal para las personas mayores" ${ }^{26}$. Por lo tanto, el objetivo de la odontogeriatría debería ser preparar a los dentistas generales para satisfacer las complejas necesidades de esta nueva población mayor.

El rápido envejecimiento poblacional a nivel mundial ha estimulado la progresiva inclusión de la odontogeriatría en el plan de estudios de muchas escuelas dentales. En los últimos treinta años, varios estudios han informado sobre el estado de la odontogeriatría en el pregrado de las escuelas de Odontología en los Estados Unidos de Norteamérica $^{27}$ y Europa ${ }^{28}$. Además, se han publicado las directrices curriculares y el contenido de los 
cursos y programas en odontogeriatría ${ }^{29}$, junto con la identificación de las competencias básicas en el área ${ }^{25}$, buscando así consenso entre las distintas entidades formadoras en un perfil común y mínimo de competencias para el manejo odontológico de las personas mayores.

Los resultados de un estudio destinado a evaluar el estado actual de la educación en odontogeriatría en Chile, con el universo de las escuelas de Odontología del país ${ }^{30}$, mostraron que sólo 37\% de ellas contaba con un curso específico de odontogeriatría. El resto de las escuelas se distribuía en diferentes tipos de metodologías de enseñanza. A pesar de contar con un bajo porcentaje de formación específica en el área, estos cursos eran impartidos alineándose a los contenidos curriculares internacionales. En general, los cursos están enfocados en la atención de la población autovalente, la cual se considera debiese ser entregada en forma obligatoria a través de la formación de pregrado ${ }^{25}$. Por su parte, la atención de la población dependiente requiere de competencias más complejas, que deberían ser abordadas en la formación de postgrado ${ }^{31}$, situación que al menos en nuestro país aún no es reconocida como una especialidad. Pese a ello, ya existen al menos tres programas de diplomado en el área y un programa de magíster profesional que se dictará a partir del año 2016. Una situación muy diferente es la que ocurre en numerosos países como Estados Unidos de Norteamérica, Nueva Zelanda, Australia, Reino Unido, países escandinavos, Brasil, Argentina, Irlanda, Japón, México, los Países Bajos y España ${ }^{32}$ donde ya la formación de postgrado es una realidad.

\section{Calidad de vida asociada a salud bucal}

Se ha demostrado, principalmente en personas mayores, que la salud bucal es un predictor de la calidad de $v i^{33}$. La autopercepción que tiene el paciente sobre su salud bucal, y el impacto que ella genera en su calidad de vida, está ampliamente reconocido como un componente importante de la salud ${ }^{34}$. El concepto de calidad de vida asociado a salud bucal es concebido como una evaluación multidimensional, auto-reportada, que mide el impacto de las condiciones bucales sobre las actividades de la vida diaria. Es cada vez más utilizado para evaluar la salud bucal, como guía para establecer prioridades a la hora de invertir en recursos, como indicador para medir eficacia en las intervenciones en salud bucal y los resultados de la atención odontológica en grupos de personas mayores $^{35}$.

Uno de los instrumentos más utilizados en el mundo, para evaluar calidad de vida asociada a salud bucal es el Oral Health Impact Profile $(\mathrm{OHIP})^{36}$, desarrollado inicialmente con 49 preguntas, recientemente también validado en Chile para población mayor ${ }^{37}$. Instrumentos extensos como éste, pueden no ser aplicables en algunas situaciones. Para optimizar la aplicación del OHIP-49, Slade et al. desarrollaron una versión acotada de 14 preguntas $^{38}$ que ha sido validada en muchos idiomas, incluyendo al español en la versión chilena ${ }^{39}$. Estudios realizados tanto en Chile ${ }^{37,39,49}$ como en Uruguay y Argentina ${ }^{41}$ han reportado una mala salud bucal agravada por una mala calidad de vida, asociada a desigualdades en educación y nivel socioeconómico.

\section{La experiencia del desarrollo de la odontogeriatría en el Maule}

Frente a la transición demográfica que rápidamente está viviendo nuestro país y a los pobres índices de salud bucal en la población mayor, el país requiere nuevo conocimiento especializado en el área de la odontogeriatría. A partir de una mirada a los centros de educación superior chilenos, es posible visualizar un desarrollo muy incipiente de la investigación en el área. En ese escenario, un grupo de académicos de la Escuela de Odontología de la Universidad de Talca decidió abordar esta temática desde la perspectiva de la Odontología. Es así que en el año 2009 se creó el Grupo de Investigación en Odontogeriatría (GIOG), cuya misión es generar investigación en odontogeriatría a nivel nacional e internacional y promover el desarrollo de la disciplina. Su visión es convertirse en un referente nacional para la investigación odontogeriátrica y contribuir como ente de vinculación para los diferentes grupos nacionales, con un importante componente de internacionalización, de suerte tal de articular investigadores en el área, principalmente en Latinoamérica. Los simposios internacionales de odontogeriatría organizados en Chile por este grupo han servido para difundir la problemática de la salud bucal de las personas mayores y crear redes de contacto para diferentes 
profesionales de la salud, no sólo odontólogos. Uno de los primeros productos de estos simposios, fue la "Declaración de Talca, 2010", que analizó la situación actual de la salud bucal de las personas mayores junto con los desafíos y estrategias a abordar para los años venideros.

Otras de las actividades de extensión del grupo ha sido la creación del Grupo Latinoamericano de Investigación en Odontogeriatría (GLIOG) dependiente de la International Association for Dental Research (IADR) que ha permitido colaborar en proyectos de investigación a nivel regional potenciando los vínculos con investigadores en varios países. En 2013, el grupo se hace parte del Programa de Investigación de Excelencia en Envejecimiento Saludable (PIEI-ES), con el fin de abordar interdisciplinariamente la investigación en la temática del envejecimiento. Entre las líneas de investigación del PIEI-ES, la salud bucal de las personas mayores constituye uno de sus focos centrales, estudiando aspectos microbiológicos, de alimentación y funcionales del adulto mayor, con estudios de ciencia básica experimentales, ensayos clínicos controlados y epidemiológicos en estas materias.

\section{Conclusiones}

El rápido envejecimiento de la población y sus aún escasamente abordadas consecuencias médicas, legales, políticas y sociales, son una realidad que demanda acciones concretas. En materia de salud bucal, este fenómeno demográfico impacta fuertemente a la población mayor que posee más dientes, pero que dada la alta prevalencia y severidad de las patologías bucales, ve afectada su calidad de vida, comprometiendo un envejecimiento saludable. Se hace evidente, por tanto, la necesidad de contar con odontólogos formados en el pregrado para la atención de la población autovalente y a nivel de postgrado, de especialistas que puedan cubrir las demandas de la población mayor dependiente. Lamentablemente, el currículo actual en la mayoría de las universidades genera profesionales que no son competentes en brindar los servicios necesarios para las demandas de la población adulta mayor. Junto con ello, las políticas actuales para la provisión de servicios odontológicos resultan claramente insuficientes, pensando en adultos mayores con expectativas de vida mayores y para los cuales la seguridad social no contempla la prestación de servicios especializados.

La formación de profesionales odontólogos en el área de la odontogeriatría debe plantearse con una mirada interdisciplinaria, donde se incorporen aquellos académicos con experiencia desde las distintas disciplinas, como la geriatría, gerontología, antropología y sociología, entre otras; que permitan una provisión de servicios integrales para los adultos mayores, apuntando a mejorar su calidad de vida.

Se espera que para el 2025, Chile se convierta en el país más envejecido de Latinoamérica y por ende su misión debiera ser el liderar cambios en la educación, en la planificación de una política de salud bucal para esta población y en la implementación de programas innovadores acorde a los avances en la investigación, no sólo de los nuevos profesionales odontólogos, sino que la de los profesionales de las distintas disciplinas de la salud. Si se materializan los esfuerzos de los distintos grupos involucrados junto con una política de Estado decidida, Chile puede llegar a convertirse en referente y modelo para enfrentar estos desafíos.

\section{Referencias}

1. ONS. Statistical bulletin: National Population Projections, 2012-based Statistical Bulletin. Available at: http://www.ons.gov.uk/ons/rel/npp/national-population-projections/2012-based-projections/stb-2012based-npp-principal-and-key-variants.html accessed: March 25, 2014. 2013.

2. Oeppen J, Vaupel JW. Demography. Broken limits to life expectancy. Science 2002; 296 (5570): 1029-31.

3. CSIS. El Desafío del Envejecimiento en América Latina. Available at: http://csis.org/files/media/csis/ pubs/090324_gai_spanish.pdf accessed: March 25, 2014. 2009.

4. CEPAL. Observatorio demográfico 2012. Proyecciones de población. Available at: http://www.eclac.cl/publicaciones/xml/1/50561/ObservatorioDemografico2012.pdf. Last accessed: March 25, 2014. 2013.

5. SENAMA. Política Integral de Envejecimiento Positivo para Chile 2012-2025. Available at: http://www.senama. cl/filesapp/PIEP-2012-2025.pdf Last accessed: March 24, 2014. 2012.

6. MINSAL. Guía Clínica: Salud Oral Integral del Adulto de 60 años. Avalilable at: http://web.minsal.cl/portal/ 
url/item/7221747c2c9484b7e04001011f0141a4.pdf Last accessed: March 25, 2014. 2010.

7. Mariño RJ, Fu CS, Giacaman RA. Prevalence of root caries among ambulant older adults living in central Chile. Gerodontology 2015; 32: 107-14.

8. Rodrigues HL, Scelza MF, Boaventura GT, Custódio SM, Moreira EA, Oliveira De L. Relation between oral health and nutritional condition in the elderly. J Appl Oral Sci. 2012; 20 (1): 38-44.

9. Mealey BL, Ocampo GL. Diabetes mellitus and periodontal disease. Periodontol 2000. 2007; 44: 127-53.

10. Syrjälä AM, Ylöstalo P, Hartikainen S, Sulkava R, Knuuttila M. Number of teeth and selected cardiovascular risk factors among elderly people. Gerodontology 2010; 27 (3): 189-92.

11. Puisieux F, D'Andrea C, Baconnier P, Bui-Dinh D, Castaings-Pelet S, Crestani B, et al. Swallowing disorders, pneumonia and respiratory tract infectious disease in the elderly. Rev Mal Respir 2011; 28 (8): e76-93.

12. Douglass CW, Shih A, Ostry L. Will there be a need for complete dentures in the United States in 2020? J Prosthet Dent 2002; 87 (1): 5-8.

13. Murray Thomson W. Epidemiology of oral health conditions in older people. Gerodontology 2014; 31 Suppl 1: 9-16.

14. Brothwell DJ, Jay M, Schönwetter DJ. Dental service utilization by independently dwelling older adults in Manitoba, Canada. J Can Dent Assoc 2008; 74 (2): 161f.

15. Ettinger RL, Mulligan R. The future of dental care for the elderly population. J Calif Dent Assoc 1999; 27 (9): 687-92.

16. Ettinger RL. A 30-year review of a geriatric dentistry teaching programme. Gerodontology 2012; 29 (2): e1252-60.

17. Chalmers JM, Carter KD, Spencer AJ. Caries incidence and increments in community-living older adults with and without dementia. Gerodontology 2002; 19 (2): 8094.

18. Guggenheimer J, Moore PA. Xerostomia: etiology, recognition and treatment. J Am Dent Assoc 2003; 134 (1): 61-9; quiz 118-9.

19. Krall E, Hayes C, García R. How dentition status and masticatory function affect nutrient intake. J Am Dent Assoc 1998; 129 (9): 1261-9.

20. Osterberg T, Carlsson GE, Sundh V, Mellström D. Number of teeth-a predictor of mortality in 70-year-old subjects. Community Dent Oral Epidemiol 2008; 36 (3): 258-68.

21. MINSAL. Encuesta Nacional de Salud. Disponible en: http://epi.minsal.cl/epi/html/invest/ENS/InformeFinalENS.pdf (Acceso el 6 de febrero de 2015).
22. MINSAL. Encuesta Nacional de Salud. Disponible en: http://epi.minsal.cl/wp-content/uploads/2012/07/ Informe-ENS-2009-2010.-CAP-5_FINALv1julioccepi. pdf. (Acceso el 6 de febrero de 2015).

23. Mariño R, Giacaman RA. Factors related to unmet oral health needs in older adults living in Chile. Arch Gerontol Geriatr 2014; 58 (3): 454-9.

24. Shinsho F. New strategy for better geriatric oral health in Japan: 80/20 movement and Healthy Japan 21. Int Dent J 2001; 51 (3 Suppl): 200-6.

25. Kossioni A, Vanobbergen J, Newton J, Müller F, Heath R. European College of Gerodontology: undergraduate curriculum guidelines in gerodontology. Gerodontology. 2009; 26 (3): 165-71.

26. Mohammad A. Geriatric dentistry: a clinical guidebook. 2nd ed. Columbus: Ohio State University. 2001.

27. Mohammad AR, Preshaw PM, Ettinger RL. Current status of predoctoral geriatric education in U.S. dental schools. J Dent Educ 2003; 67 (5): 509-14.

28. Nitschke I, Kunze J, Reiber T, Sobotta BA. Development of undergraduate gerodontology courses in Austria, Switzerland, and Germany from 2004 to 2009. J Dent Educ 2013; 77 (5): 630-9.

29. Curriculum guidelines for geriatric dentistry. J Dent Educ 1989; 53 (5-6): 313-6.

30. León S, Araya-Bustos F, Ettinger RL, Giacaman RA. Geriatric dentistry content in the curriculum of the dental schools in Chile. Gerodontology 2014 Nov 30. doi: 10.111/ger.12171.

31. Dolan T, Berkey D, Mulligan R, Saunders M. The state of the art of geriatric dental education and training. In: Klein SM (editor). A National Agenda for geriatric education: white papers. New York: Springer Publishing Co. 1995; p. 125-53.

32. MacEntee MI. How should we educate dental geriatricians? Gerodontology 2013; 30 (2): 89-90.

33. Petersen PE, Kandelman D, Arpin S, Ogawa H. Global oral health of older people-call for public health action. Community Dent Health 2010; 27 (4 Suppl 2): 257-67.

34. John MT, Patrick DL, Slade GD. The German version of the Oral Health Impact Profile-translation and psychometric properties. Eur J Oral Sci 2002; 110 (6): 425-33.

35. Sanders AE, Slade GD, Lim S, Reisine ST. Impact of oral disease on quality of life in the US and Australian populations. Community Dent Oral Epidemiol 2009; 37 (2): 171-81.

36. Slade GD, Spencer AJ. Development and evaluation of the Oral Health Impact Profile. Community Dent Health 1994; 11 (1): 3-11.

37. León S, Bravo-Cavicchioli D, Giacaman RA, Correa-Bel- 
trán G, Albala C. Validation of the Spanish version of the oral health impact profile to assess an association between quality of life and oral health of elderly Chileans. Gerodontology 2014 Mar 11. doi; 10.1111/ger.12124.

38. Slade GD. Derivation and validation of a short-form oral health impact profile. Community Dent Oral Epidemiol 1997; 25 (4): 284-90.

39. León S, Bravo-Cavicchioli D, Correa-Beltrán G, Giacaman RA. Validation of the Spanish version of the Oral
Health Impact Profile (OHIP-14Sp) in elderly Chileans. BMC Oral Health 2014; 14: 95.

40. Mariño R, Albala C, Sánchez H, Cea X, Fuentes A. Self-assessed oral-health status and quality of life of older Chilean. Arch Gerontol Geriatr 2013; 56 (3): 513-7.

41. Fuentes-García A, Lera L, Sánchez H, Albala C. Oral health-related quality of life of older people from three South American cities. Gerodontology 2013; 30 (1): 67 75. 